INPLASY

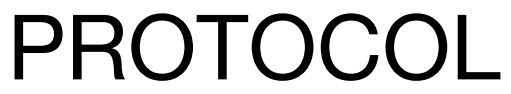

To cite: Feng et al. Traditional Chinese medicine intervention for autism spectrum disorders: A protocol for systematic review and network metaanalysis. Inplasy protocol 202210137. doi:

10.37766/inplasy2022.1.0137

Received: 31 January 2022

Published: 31 January 2022

Corresponding author: Jiangshan Li

001316@hnucm.edu.cn

Author Affiliation:

School of Acupuncture-

Moxibustion and Tuina, Hunan

University of Chinese

Medicine.

Review Stage at time of this submission: The review has not yet started.

Conflicts of interest:

None declared.

\section{Traditional Chinese medicine intervention for autism spectrum disorders: A protocol for systematic review and network meta-analysis}

Feng, X1; Li, KS2; Jiang, QR 3; Zhang, YX4; Gong, ZC5; Zhi, H6; Yu, J7; Li, W8; Li, JS9.

Review question / Objective: The purpose of this study was to evaluate the effectiveness and safety of conventional treatment-based TCM interventions for ASD.

Eligibility criteria: The study will focus on all complete randomized controlled trials of TCM interventions in ASD, and subjects from any ethnic background will be included in the study. However, language will be limited to studies published in English or Chinese only, and non-randomized controlled trials (e.g., sequential trials, systematic reviews, case reports, clinical experiences, conference abstracts, animal studies, cellular studies) will not be considered.

INPLASY registration number: This protocol was registered with the International Platform of Registered Systematic Review and Meta-Analysis Protocols (INPLASY) on 31 January 2022 and was last updated on 09 February 2022 (registration number INPLASY202210137).

\section{INTRODUCTION}

Review question / Objective: The purpose of this study was to evaluate the effectiveness and safety of conventional treatment-based TCM interventions for ASD.
Condition being studied: Autism spectrum disorder is a pervasive neurodevelopmental disability, and the DSM-5 (Diagnostic and statistical manual of mental disorders, fifth edition) specifies its main core symptoms like impairments in social communication, social interaction, and restrictive or 
repetitive behaviors. In addition to the two core symptoms, individuals with ASD have some impairments in cognitive functioning and affective behavior.In recent years, the prevalence of autism has increased gradually, and the Centers for Diseases and Control Prevention(CDC) released the most recent data on the prevalence of autism at the end of 2021, showing that 1 in 44 children are diagnosed with ASD.The cause of ASD has not been identified but is now widely believed to be the result of the interaction between environmental and genetic factors.As research has progressed, rare variants in genes, as well as common genetic variants with small effects, have been shown to increase the risk of developing ASD. Children with ASD have difficulties in social integration, and some of the core symptoms remain for the rest of their life, requiring long-term rehabilitation. The Lancet pointed out that patients with severe ASD currently account for approximately $30 \%$ of all ASD patients and that they are in need of more treatment and rehabilitation opportunities. The high cost of rehabilitation and medical expenses puts a heavy burden on society and ASD families. There is no specific treatment for the rehabilitation of ASD, but the main focus of current treatments is on behavioral interventions and physical therapy. Applied Behavior Analysis (ABA) is a behavioral training model that uses a stimulus-response-reinforcement approach with the goal of developing socially adaptive behaviors, reducing problematic behaviors, and emphasizing parental involvement. Physiotherapy includes transcranial magnetic stimulation, which is often preferred, because of its painlessness and low side effects, to reduce repetitive behaviors and sensitivity to the environment in patients. In contrast, pharmacological treatment is not currently a common intervention, but it has a certain alleviating effect on some ASD symptoms. All of the above treatments have contributed to the recovery of children with ASD. Traditional Chinese Medicine (TCM) has been guarding the health of the Chinese people for thousands of years and has been used to treat diseases through clinical practice using herbal medicines in formulas or external treatments, such as acupuncture, moxibustion, Tuina, and Guasha. However, because of historical reasons, there is a lack of evidence of

\section{METHODS}

Search strategy: Starting in January 2022, we will conduct comprehensive searches in the following databases: China Biological Medicine Database, Chinese Scientific Journals Database, Wan Fang databases, and China National Knowledge Infrastructure, the Cochrane Library, Web of Science, PubMed, EMBASE Database.

Participant or population: Due to the specific nature of ASD and its difficulties in early diagnosis, the age range of patients with ASD included in the study was 2-18 years, thus ranging from early childhood to adolescence. All participants, regardless of ethnic background and sex, were diagnosed with ASD according to relevant guidelines or consensus.

Intervention: Traditional Chinese medicine.

\section{Comparator: Conventional intervention.}

Study designs to be included: Randomized controlled trials of TCM interventions in ASD.

Eligibility criteria: The study will focus on all complete randomized controlled trials of TCM interventions in ASD, and subjects from any ethnic background will be included in the study. However, language will be limited to studies published in English or Chinese only, and nonrandomized controlled trials (e.g., sequential trials, systematic reviews, case reports, clinical experiences, conference abstracts, animal studies, cellular studies) will not be considered.

Information sources: China Biological Medicine Database, Chinese Scientific Journals Database, Wan Fang databases, and China National Knowledge Infrastructure, the Cochrane Library, Web of Science, PubMed, EMBASE Database. 
Main outcome(s): The main efficacy evaluation index is based on the beforeand-after results at the end of the intervention or at the end of the follow-up period to evaluate the effectiveness of the intervention. The efficacy, effectiveness, and ineffectiveness will be evaluated according to the relevant forms or questionnaires, which are based on research tools commonly used in clinical practice, including the ADOS (Autism Diagnostic Observation Scale), ADI-R (Autism Diagnostic Interview-Revised), and CARS (Childhood Autism Rating Scale). It is worth mentioning that even one of these questionnaires is sufficient for evaluation, considering their limited availability (in particular ADOS and ADI-R) in China due to copyright restrictions.

Additional outcome(s): Additional outcomes will include behavioral questionnaires, such as the autism behavior checklist (ABC) and autism treatment evaluation checklist (ATEC), to evaluate the effect of TCM on the improvement of ASD symptoms in multiple dimensions, and may also include some symptom score scales

Quality assessment / Risk of bias analysis: Quality assessment will be conducted by two researchers (Yuxing Zhang and Hui Zhi) to independently assess the risk of bias in all included randomized controlled trials using the Cochrane Collaboration tool, which focuses on the following aspects: task concealment, random sequence generation, blinding of outcome assessors, participant and personnel blinding, selective reporting, completeness of outcome data, and other sources of bias. Each domain was classified as high-risk, low-risk, or unclear risk. Should disagreements arise during the study, they will be resolved through discussion with a third senior assessor (Wu Li).

Strategy of data synthesis: First, for the direct comparisons, a conventional metaanalysis will be performed using Revman 5.3. (Cochrane Collaboration, Oxford, UK). Second, considering the anticipated heterogeneity, the network meta-analysis within a Bayesian framework will be conducted using WinBUGS 1.4.3 (MRC Biostatistics Unit, Cambridge, UK) based on the random effect model for the results of the indirect comparison. In addition, models will be calculated using the Markov chain Monte Carlo algorithm: four chains will be employed for simulation analysis, the step size will be set to 10 , the number of annealing times will be set to 20,000 to reduce the impact on arbitrary values, and the number of iterations will be set to 50,000 . Additionally, continuous outcomes will be measured by standard mean difference with $95 \%$ confidence intervals for indirect comparisons.Third, the plot of the surface under the cumulative ranking curve will be computed using STATA 14.0. (Stata Corporation, College Station, TX) to forecast the possible ranking order. In our study, a higher surface under the cumulative ranking curve score represents a better TCM intervention for ASD.

Subgroup analysis: Not mentioned.

Sensitivity analysis: In general, differences between different types of evidence may be the main reason for the inconsistency. Therefore, inconsistencies between indirect and direct evidence will be assessed using circular inconsistency tests and nodal splitting methods. In addition, Zvalues and the corresponding $P$-values will be calculated, with $P$-values less than 0.05 indicating significant differences. If significant heterogeneity is found, subgroup analyses will be performed according to possible sources of heterogeneity, such as the severity of ASD in the children, age stratification, and evaluation forms.

Language: Chinese and English.

Country(ies) involved: China.

Keywords: autism spectrum disorders, network meta-analysis, protocol, systematic review, Traditional Chinese medicine. 
Contributions of each author:

Author 1 - Xiang Feng - Writing - original draft,Conceptualization,Formal analysis.

Email: 20173147@stu.hnucm.edu.cn

Author 2 - Keshang Li - Conceptualization.

Author 3 - Quanrui Jiang - Data curation.

Author 4 - Yuxing Zhang - Data curation.

Author 5 - Zhichao Gong - Data curation.

Author 6 - Hui Zhi - Formal analysis.

Author 7 - Jun Yu.

Author 8 - Wu Li - Methodology.

Author 9 - Jiangshan Li - corresponding author, Supervision.

Support: This research was supported by grants from the Research and Development Program in Key Areas of Hunan Province (No. 2019SK2081), Research and Innovation Project for Graduate Students in Hunan Province (No. CX2018B487), Science and Technology Project of Changsha City (No. kq1901093), and Research and Innovation Project for Graduate Students of Hunan University of Chinese Medicine (No. 2021CX15).

Author Affiliation: 1.School of AcupunctureMoxibustion and Tuina, Hunan University of Chinese Medicine, Changsha, Hunan, China.

2.College of Acupuncture and Orthopedics, Hubei University of Chinese Medicine, Wuhan, Hubei, China.

3.The first affiliated hospital of Hunan University of Chinese Medicine,Changsha, Hunan, China. 